\section{OPEN JOURNAL SYSTEMS}

ISSN:2237-2202
Available on line at Directory of Open Access Journals

Journal of Hyperspectral Remote Sensing v.9, n.4 (2019) 191-203

www.periodicos.ufpe.br/revistas/jhrs
Journal of Hyperspectral Remote Sensing

www.ufpe.br/jhrs

\title{
Geotechnology in the analysis of behavior spectral of natural resources in the Semiarid pernambucano
}

\author{
Haylla R. de A. L. Leonardo ", Leidjane M. M. de Oliveira**, Estefany F. de Oliveira ${ }^{* * *}$, Débora N. O. \\ de Almeida ${ }^{* * * *}$, Anderson L. R. de Paiva \\ ${ }^{*}$ Graduanda, Departamento de Engenharia Civil e Ambiental, Universidade Federal de Pernambuco (UFPE), Recife-PE, Brasil. E- \\ mail: hayllarebeka.hr@gmail.com (autor correspondente) \\ ***Professora D.Sc. do Departamento de Engenharia Civil e Ambiental e da Pós-Graduação em Ciências Geodésicas e Tecnologia \\ da Geoinformação, UFPE, Recife-PE, Brasil. E-mail: leidjaneoliveira@ hotmail.com \\ *** Graduanda, Departamento de Engenharia Civil e Ambiental, UFPE, Recife-PE, Brasil. E-mail: estefanyferreira10@gmail.com \\ **** Mestranda da Pós-Graduação em Ciências Geodésicas e Tecnologias da Geoinformação, UFPE, Recife-PE, Brasil. E-mail: \\ debora.nathalia21@gmail.com; \\ ******Professor D.Sc. do Departamento de Engenharia Civil e Ambiental e da Pós-Graduação em Engenharia Civil, UFPE, Recife- \\ PE, Brasil. E-mail: anderson.paiva@ufpe.br.
}

Received 22 August 2019; accepted 30 October 2019

\begin{abstract}
The study of soil cover in the wilderness of the Pernambuco State, through georeferenced images obtained by remote sensing, is of great relevance in the observation of the region as the spectral variability of the vegetation and the use and occupation of the soil. This study aimed to evaluate the NDVI (Normalized Difference Vegetation Index) and NDWI (Normalized Difference Water Index) index in the municipalities of Mirandiba, Carnaubeira da Penha and Bélem do São Francisco. Were acquired the TM Landsat 5 images from the study region referring to date of November 9, 2005 and for OLI Landsat 8 sensor the date corresponding to November 13, 2018. With the processing of these images using the free software QGIS, we found values of NDVI in the interval referring to values smaller than 0.10, at 0.60, NDWI between negative values at 0.20 , being thus characterized the situation of exposed soil and sparse vegetation. The mapping of the surface area of the Pajeú river was carried out in the region of the Serrinha dam, obtaining the visualization of the water body variation during the 13 years, and the classification of the areas around this region using the project of Brazil's Annual Mapping of Soil Coverage and Land Use (MAPBIOMAS).
\end{abstract}

Keywords: vegetação, TM Landsat 5, OLI Landsat 8.

\section{Geotecnologia na análise do comportamento espectral dos recursos naturais no Semiárido pernambucano}

\section{Resumo}

O estudo da cobertura do solo no sertão de Pernambuco, através de imagens georreferenciadas obtidas por Sensoriamento Remoto, é de suma relevância na observação da região quanto à variabilidade espectral da vegetação e do uso e ocupação do solo. Neste estudo objetivou-se em avaliar os índices NDVI (Índice de Vegetação por Diferença Normalizada) e NDWI (Índice de Água por Diferença Normalizada), nos municípios de Mirandiba, Carnaubeira da Penha e Belém do São Francisco. Foram adquiridas as imagens TM Landsat 5 da região de estudo referente a data 09 de novembro de 2005 e para sensor OLI Landsat 8 a data correspondente a 13 de novembro de 2018. Com o processamento dessas imagens utilizando o software de livre acesso QGIS, encontraram-se valores de NDVI no intervalo referente a valores menores de 0,10 a 0,60; NDWI entre valores negativos a 0,20, sendo assim caracterizada a situação de solo exposto e vegetação escassa. Foi realizada a geração do mapa da área superficial do rio Pajeú, na região da barragem de Serrinha, obtendo-se a visualização da variação do corpo hídrico no transcorrer dos 13 anos, e a classificação das áreas no entorno desta região utilizando-se o projeto de Mapeamento Anual de Cobertura e Uso do Solo do Brasil (MAPBIOMAS).

Palavras-chave: vegetação, TM Landsat 5, OLI Landsat 8. 


\section{Introdução}

Atualmente o monitoramento do comportamento hídrico associado a variação da cobertura vegetal a partir de técnicas de Sensoriamento Remoto (SR) encontra-se cada vez mais recorrente no cenário mundial (Farias et al., 2017). Várias aplicações têm sido feitas para o Sertão nordestino, que é caracterizado por longos períodos de estiagem, provocando um cenário de grande estresse hídrico e, consequentemente vegetal, na região, segundo Gordon et al. (2005).

Além deste fato, as atividades antrópicas de irrigação na região provocam alterações na cobertura vegetal nativa, a exemplo do cenário observado na região de estudo dos municípios de Belém de São Francisco, Carnaubeira da Penha, e Mirandiba, situados na Bacia do Pajeú e na Bacia GI4.

Através de suas ferramentas, o Sensoriamento Remoto permite a observação dos parâmetros espectrais da vegetação e parâmetros biofísicos da paisagem, resultando na disseminação de pesquisas do uso do solo devido ao fornecimento de informações atualizadas facilitando diagnósticos de ocupações e parâmetros biofísicos (Silva et al., 2019).

A aplicação das técnicas de Sensoriamento Remoto fundamenta-se na compreensão do comportamento espectral que uma dada cobertura assume em um determinado produto orbital. Esse produto é fruto de um processamento de imagens de satélite que pode envolver diversos parâmetros e fatores ambientais (Ponzoni, 2002). O uso de ferramentas de Sistema de Informação Geográficas (SIGs) e Sensoriamento Remoto facilitam a obtenção de dados de modo mais eficiente e econômico para estudos mais completos nesse âmbito (Araújo et al., 2015).

Entre diversas técnicas, destacam-se os índices de vegetação e água, através dos quais analisa-se a configuração da superfície terrestre através dos comportamentos espectrais das feições encontradas na mesma. Os índices NDVI (Normalized Difference Vegetation of Index) e NDWI (Normalized Difference Water of Index) foram analisados por Gonzaga et al. (2011), utilizando como área de estudo o município de Piranhas no estado de Alagoas. O estudo foi realizado observando os períodos do ano em que ocorreram intensidades pluviométricas diferentes, obtendo-se a premissa que durante esses períodos os índices apresentaram valores mais elevados. Silva et al. (2019) realizaram um estudo utilizando o NDVI e o NDWI para a análise temporal da cobertura do solo em um município do sertão de Pernambuco, evidenciando o aumento no valor do NDVI para períodos chuvosos, corroborando com a premissa observada por Gonzaga et al. (2011).

O MAPBIOMAS é um projeto de Mapeamento Anual de Cobertura e Uso do Solo do Brasil, que reúne uma rede colaborativa nas áreas de sensoriamento remoto, biomas, usos da terra, SIG e ciência da computação. Utiliza processamento em nuvem e classificadores automatizados, desenvolvidos e operados, a partir da plataforma Google Earth Engine que permite gerar uma série histórica de mapas anuais de cobertura e uso da terra do Brasil (MAPBIOMAS, 2019).

Almeida et al. (2018), Gomes et al. (2017) e Lima et al. (2017) apresentaram aplicações dessa ferramenta para analisar o uso e cobertura dos solos de municípios inseridos na região semiárida de Pernambuco, respectivamente, no período de 2000 a 2017.

Neste trabalho, objetivou-se utilizar a geotecnologia na análise do comportamento espectral dos recursos naturais no Semiárido pernambucano, especificamente dos municípios de Belém de São Francisco, Carnaubeira da Penha e Mirandiba, em uma perspectiva espectro temporal, para a comparação da variação entre os anos de 2005 e 2018, através do estudo dos índices NDVI, NDWI e ferramentas do projeto MAPBIOMAS da coleção 3.1 (PROJETO MAPBIOMAS, 2019).

\section{Material e métodos}

\section{Área de estudo}

A área de estudo (Figura 1) abrange os municípios da região Nordeste, no sertão do semiárido de Pernambuco, os municípios estudados foram: Mirandiba localizado nas coordenadas $08^{\circ} 07^{\prime} 13$ " de latitude sul, $38^{\circ} 43^{\prime}$ 46" de longitude oeste, composto por uma área de $773,3 \mathrm{Km}^{2}$; o segundo, Carnaubeira da Penha localizado nas coordenadas $08^{\circ} 19^{\prime} 09^{\prime}$ ' de latitude sul, $38^{\circ} 44^{\prime} 41^{\prime \prime}$ de longitude oeste, composto por uma área de 999,4 Km²; e Belém do São Francisco localizado nas coordenadas $08^{\circ} 45^{\prime} 14^{\prime \prime}$ de latitude sul, $38^{\circ} 57^{\prime} 57^{\prime}$ ' de longitude oeste, composto por uma área de $1.842,7 \mathrm{Km}^{2}$.

Foram identificados dois corpos hídricos principais na região analisada, o primeiro trata-se de uma fração do Rio São Francisco, localizado na região sul do município de Belém de São Francisco, e o segundo o Rio Pajeú, na barragem de Serrinha, localizado a leste do município de Mirandiba. 


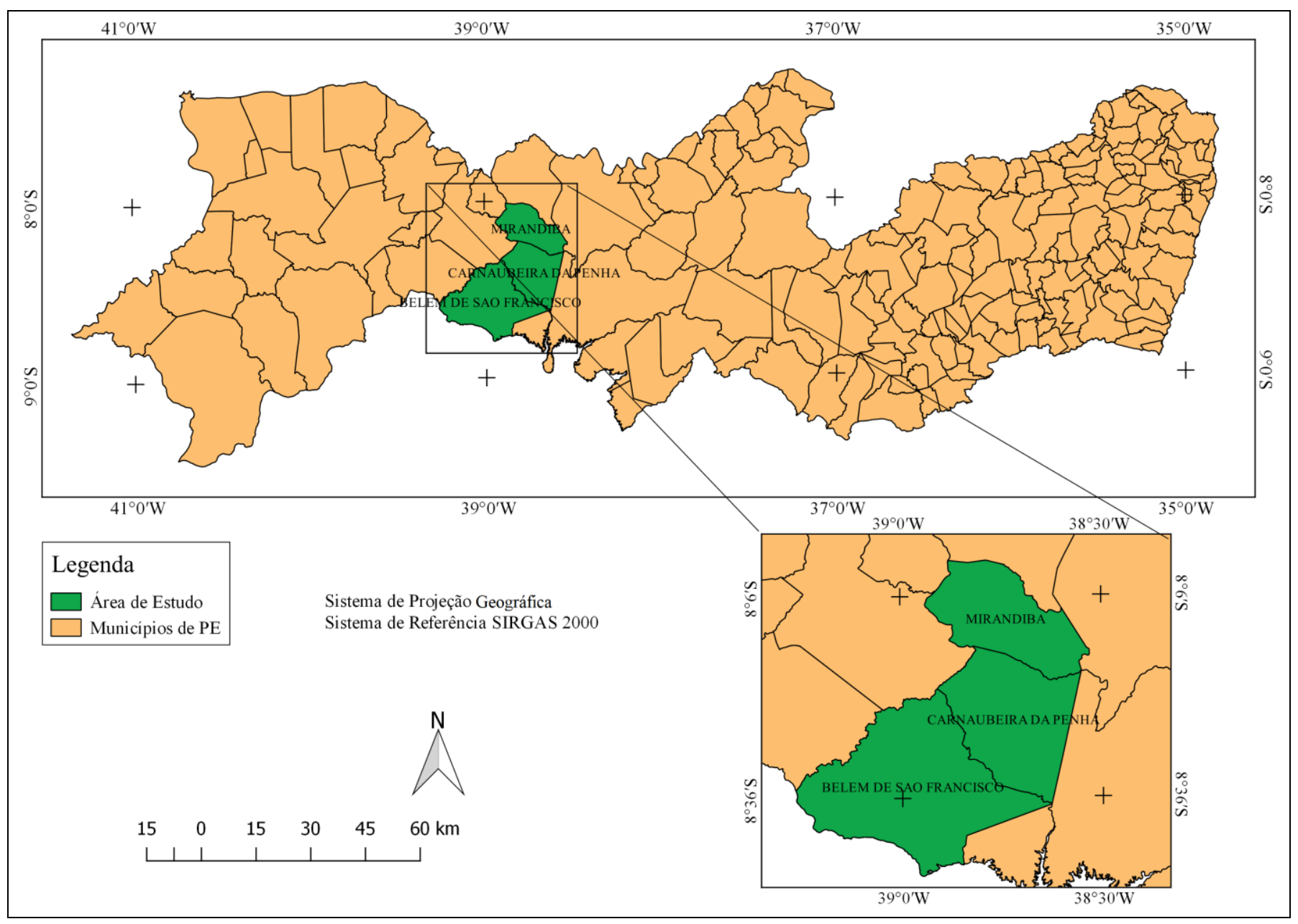

Figura 1 - Localização da área de estudo.

\section{Distribuição pluviométrica nos anos 2005 e 2018}

Para a identificação dos índices pluviométricos da área estudada utilizou-se o banco de dados obtido pela Agência Pernambucana de Águas e Clima (APAC) referentes ao posto 54, situado no município de Mirandiba - PE. Foram estudados os dados pluviométricos médios mensais do ano de 2005, referente à data da imagem TM Landsat 5, e os dados pluviométricos médios mensais do ano de 2018, referentes à data da imagem OLI Landsat 8.

Observou-se que a partir do mês de julho do ano de 2005 ocorreu um declínio no gráfico da precipitação e entre os meses de agosto e setembro não há precipitação (Figura 2).

A comparação dos dados dos anos de estudos evidenciou a ocorrência de uma maior precipitação pluviométrica em 2005 com um total de precipitação pluviométrica anual de 799,7 $\mathrm{mm}$; enquanto que para o ano de 2018 a precipitação pluviométrica anual foi de $615 \mathrm{~mm}$. Entretanto, segundo os dados pluviométricos diários obtidos no site da APAC, o evento de precipitação de $42 \mathrm{~mm}$ ocorrido no mês de novembro do ano de 2005, ocorreu no dia 28 deste mês, posterior da data da imagem processada, nos dias anteriores a este evento não houve precipitação. Com base nestes fatos ressalta-se que o volume precipitado no ano de 2005, no período anterior a imagem analisada, foi suficiente para o abastecimento dos corpos hídricos da região, no entanto, a vegetação nativa (Caatinga) responde de forma rápida aos eventos pluviométricos, de maneira que, o fato da não ocorrência de precipitação nos dois meses próximos a data referente a passagem do satélite, corroborou para o resultado obtido na vegetação da região estudada. 


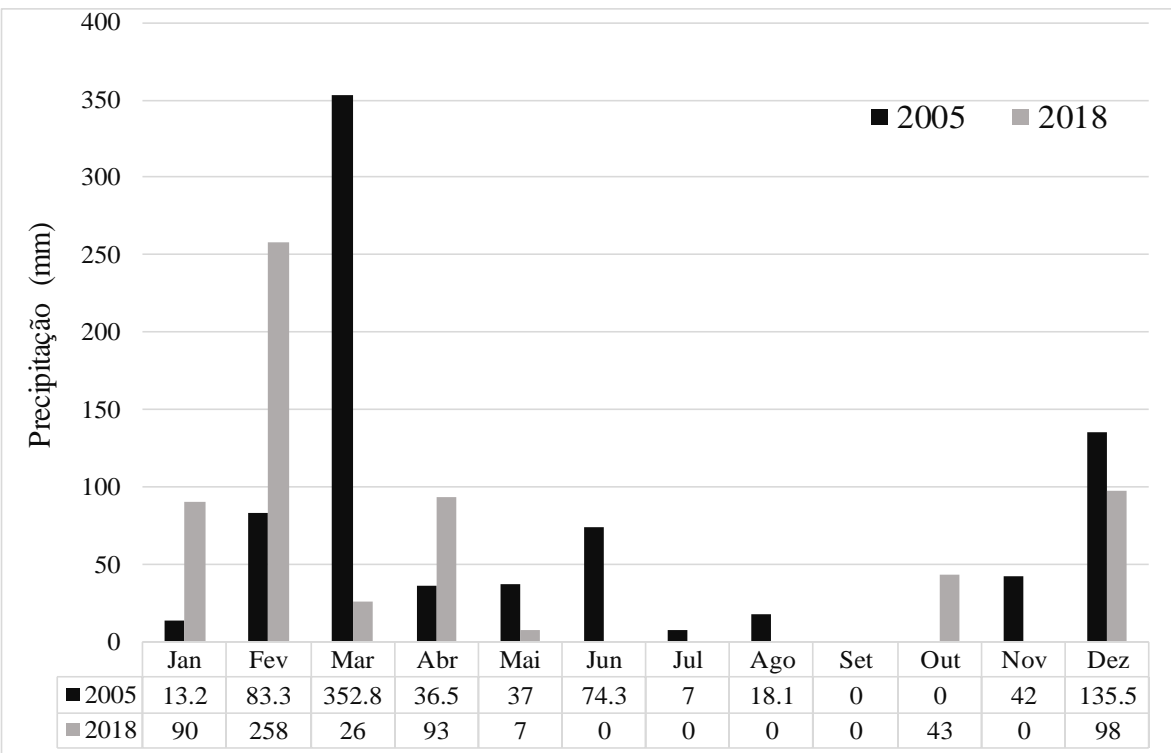

Figura 2 - Precipitação pluviométrica mensal (mm) dos anos de 2005 e 2018 do município de Mirandiba - PE. Fonte: APAC (2019).

\section{Pré-processamento}

Utilizaram-se imagens referentes ao dia 09 de novembro de 2005, do satélite Land Remote Sensing Satellite (Landsat 5), sensor Thematic Mapper (TM), órbita 216 e ponto 66, e a imagens datada em 13 de novembro de 2018, do satélite Land Remote Sensing Satellite (Landsat 8), sensor Operational Lander
Imager composta por bandas espectrais adquiridas gratuitamente através do site do United States Geological Survey (USGS), Tabela 1. As imagens orbitais e os arquivos vetoriais foram reprojetados para o Sistema Geodésico de Referência SIRGAS 2000 (Sistema de Referência Geocêntrico para as Américas), UTM fuso 24 Sul.

Tabela 1 - Data da imagem, hora, ângulo de elevação do Sol e ponto do satélite.

\begin{tabular}{ccccc}
\hline Data & Hora $($ UTM $)$ & Ângulo de elevação do Sol & Órbita & Ponto \\
\hline $09 / 11 / 2005$ & $12: 29: 40$ & 62,5359 & 216 & 66 \\
$13 / 11 / 2018$ & $12: 41: 45$ & 64,6538 & 216 & 66 \\
\hline
\end{tabular}

Fonte: USGS (2019).

Após a aquisição das imagens, realizou-se o processamento, considerando $\mathrm{o}$ recorte da cena conforme a área de interesse. Para aquisição dos parâmetros biofísicos NDVI e NDWI, utilizou-se a ferramenta "Calculadora Raster" do software QGIS versão 2.18.16. Conforme a Figura 3, foram descritas as etapas realizadas para o processamento digital das imagens para o presente estudo.

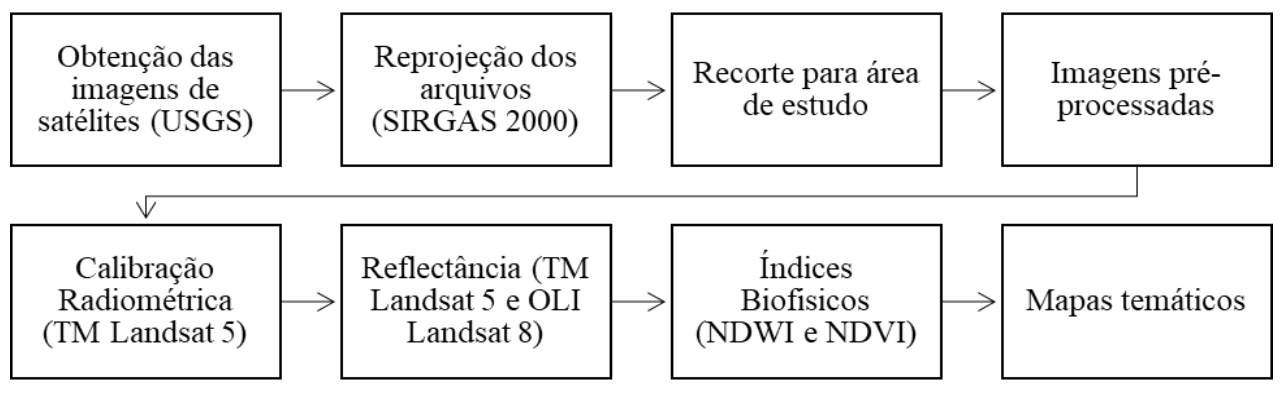

Figura 3 - Fluxograma das etapas de processamento. 
Processamento das imagens para o TM Landsat 5

Para o cálculo dos índices NDVI e NDWI foram utilizadas a radiância espectral e a reflectância das bandas 3, 4 e 5 do TM Landsat5.

Inicialmente, realizou-se a calibração radiométrica, a fim de converte o número digital (ND) em radiância espectral de cada banda por meio da equação de Markham e Baker (1987):

$$
\mathrm{L}_{\lambda \mathrm{i}}=\mathrm{a}_{\mathrm{i}}+\frac{\mathrm{b}_{\mathrm{i}}-\mathrm{a}_{\mathrm{i}}}{255} \mathrm{ND}
$$

em que:

$\mathrm{L}_{\chi_{\mathrm{i}}}$ é a radiância espectral de cada banda $\left(\mathrm{Wm}^{-2} \mathrm{sr}^{-1}\right.$ $\left.\mu \mathrm{m}^{-1}\right)$, a e b são as radiâncias espectrais mínimas e máximas de cada banda do TM Landsat 5 (Tabela 2)

Tabela 2 - Dados de comprimento de onda $(\mu \mathrm{m})$, coeficiente de calibração $\left(\mathrm{Wm}^{-2} \mathrm{sr}^{-1} \mu \mathrm{m}^{-1}\right)$ e irradiância espectral no Topo da Atmosfera $-\mathrm{K} \lambda \mathrm{i}\left(\mathrm{Wm}^{-2} \mu \mathrm{m}^{-1}\right)$ do TM Landsat5.

\begin{tabular}{|c|c|c|c|c|}
\hline \multirow[t]{2}{*}{ Bandas } & \multirow{2}{*}{$\begin{array}{l}\text { Comprimento de } \\
\text { onda }(\mu \mathrm{m})\end{array}$} & \multicolumn{2}{|c|}{$\begin{array}{l}\text { Coeficientes de Calibração } \\
\left(\mathrm{Wm}^{-2} \mathrm{sr}^{-1} \mu \mathrm{m}^{-1}\right)\end{array}$} & \multirow{2}{*}{$\begin{array}{l}\text { Irradiância espectral no } \\
\text { Topo da Atmosfera }-\mathrm{K} \lambda \mathrm{i} \\
\left(\mathrm{Wm}^{-2} \mu \mathrm{m}^{-1}\right)\end{array}$} \\
\hline & & $\mathrm{a}$ & $\mathrm{b}$ & \\
\hline 3 (V - vermelho) & $0,626-0,693$ & $-1,17$ & 264,30 & 1536 \\
\hline 4 (IV - próximo) & $0,776-0,904$ & $-1,51$ & 206,20 & 1031 \\
\hline 5 (IVM - médio) & $1,567-1,784$ & $-0,37$ & 27,19 & 220 \\
\hline
\end{tabular}

Fonte: Adaptado de Chander et al. (2009).

Sequencialmente, obteve-se a reflectância monocromática de cada banda $\left(\rho_{\lambda_{i}}\right)$, sendo esta a razão entre o fluxo de radiação refletida e o fluxo de radiação incidente, sendo calculada por meio de Allen et al. (2002):

$$
\begin{aligned}
& \rho_{\lambda \mathrm{i}}=\frac{\pi \cdot \mathrm{L}_{\lambda \mathrm{i}}}{\mathrm{K}_{\lambda_{\mathrm{i}}} \cdot \cos \mathrm{Z} \cdot \mathrm{d}_{\mathrm{r}}} \\
& \cos \mathrm{Z}=\cos \left(\frac{\pi}{2}-\mathrm{E}\right) \\
& \mathrm{d}_{\mathrm{r}}=1+0,033 \cos \left(\frac{\mathrm{DSA} \cdot 2 \pi}{365}\right)
\end{aligned}
$$

em que:

$\rho_{\lambda_{\mathrm{i}}}$ é a reflectância monocromática de cada banda, $\mathrm{L}_{\lambda_{\mathrm{i}}}$ é a radiância espectral, $\mathrm{K}_{\lambda_{\mathrm{i}}}$ é a irradiância solar espectral no topo da atmosfera, $\mathrm{Z}$ é o ângulo zenital e $\mathrm{d}_{\mathrm{r}}$ a distância Terra-sol.

O NDVI (Índice de Vegetação por Diferença Normalizada) foi calculado por meio da equação de Rouse et al. (1973) podendo variar entre +1 e -1, sendo valores positivos indicadores de região com vegetação e valores negativos indicadores de região com água ou nuvens.

$O$ índice de vegetação NDVI permite a observação das mudanças na cobertura vegetal, possibilitando a construção de um perfil temporal de crescimento. Sendo calculado em função da reflectância da banda do infravermelho próximo
(Banda 4 - $\rho \mathrm{IV}$ ) e a reflectância da banda do vermelho (Banda $3-\rho V$ ), de acordo com a equação a seguir:

$$
\mathrm{NDVI}=\frac{\left(\rho_{I V}-\rho_{V}\right)}{\left(\rho_{I V}+\rho_{V}\right)}
$$

O NDWI (Índice de Água por Diferença Normalizada), proposto por GAO (1996), permite detectar com maior qualidade a mudança da biomassa vegetal no que concerne a quantidade de água em culturas agrícolas, pela configuração do estresse hídrico no ambiente, por meio da razão entre as bandas do infravermelho próximo (Banda 4 - $\rho I V$ ) e do infravermelho médio (Banda 5 - $\rho \mathrm{IVM}$ ), de acordo com a equação:

$$
\mathrm{NDWI}=\frac{\rho_{\mathrm{IV}}-\rho_{\mathrm{IVM}}}{\rho_{\mathrm{TV}}+\rho_{\mathrm{TVM}}}
$$

\section{Processamento das imagens para o OLI Landsat 8}

$\mathrm{O}$ processamento das imagens Landsat $8 \mathrm{OLI}$ se deu em conformidade com Silva et al. (2015): para conversão dos valores quantizados e calibrados (ND) do sistema sensor Landsat 8 OLI para reflectância espectral, utilizou-se coeficientes radiométricos disponibilizados no arquivo de metadados das imagens e informações de características do sensor (Tabela 3), USGS (2019). 
Tabela 3 - Dados de comprimento de onda $(\mu \mathrm{m})$ e resolução espacial (m x m) das imagens do OLI Landsat8.

\begin{tabular}{ccc}
\hline Bandas & Comprimento de onda $(\mu \mathrm{m})$ & Resolução Espacial $(\mathrm{m} \times \mathrm{m})$ \\
\hline 4 (Vermelho) & $0,640-0,670$ & 30 \\
5 (IV - Próximo) & $0,850-0,880$ & 30 \\
6 (IVM - Médio) & $1,570-1,650$ & 30 \\
\hline
\end{tabular}

Fonte: USGS (2019).

Para a reflectância planetária no topo da atmosfera $(\rho \lambda)$, corrigida em função do ângulo solar e da distância astronômica Terra-Sol (d) foi usada a Equação 7. Mp corresponde ao fator multiplicativo de reescalonamento para cada banda (disponível nos metadados da imagem), Qcal o número digital para cada pixel e A $\rho$ corresponde ao fator aditivo de reescalonamento para cada banda (disponível nos metadados da imagem).

$\rho \lambda=\frac{M \rho * Q c a l+A \rho}{\cos (\theta s z) \frac{1}{d^{2}}}$

em que: $\rho \lambda$ corresponde a reflectância planetária no topo da atmosfera corrigida, $\theta$ se corresponde ao ângulo de elevação solar (disponível nos metadados da imagem) e $\theta$ sz corresponde ao ângulo zenital solar local (calculado a utilizando a expressão $\theta \mathrm{sz}=90^{\circ}-$ $\theta$ se).

O cálculo do NDVI se deu em conformidade com a Equação 8 utilizando as bandas do vermelho $\left(\rho_{\mathrm{V}}\right)$ e infravermelho próximo $\left(\rho_{\mathrm{IV}}\right)$, resultantes da reflectância corrigida.

$\mathrm{NDVI}=\frac{\left(\rho_{\mathrm{IV}}-\rho_{\mathrm{V}}\right)}{\left(\rho_{\mathrm{IV}}+\rho_{\mathrm{V}}\right)}$

Para o cálculo do NDWI foram utilizadas as bandas do infravermelho próximo ( $\rho_{\text {IV }}$ ) e infravermelho médio $\left(\rho_{\mathrm{IVM}}\right)$ da reflectância corrigida por meio da Equação 9 anteriormente citada.

$\mathrm{NDWI}=\frac{\left(\rho_{\mathrm{IV}}-\rho_{\mathrm{IVM}}\right)}{\left(\rho_{\mathrm{IV}}+\rho_{\mathrm{IVM}}\right)}$

\section{Resultados e discussão}

Evidenciou-se que o NDVI da região de estudo observado na Figura 4, permite a visualização da atividade ocorrida na vegetação, ressaltando-se que para os picos verdes, regiões onde o valor do NDVI é superior a 0,600 , há a ocorrência de maior atividade de vegetação.

A comparação entre o NDVI obtido pelo processamento dos dados dos anos de 2005 e 2018, foi realizada com a finalidade de observação da variação espectro temporal do índice, de maneira a se utilizar para a compreensão da mudança ocorrida na cobertura vegetal da região no decorrer dos anos.

Segundo Chouhan e Rao (2011), para o NDVI, valores muito baixos (menores que 0,1) correspondem a áreas estéreis de rocha ou areia. Valores moderados representam arbustos e pastagens $(0,2$ a 0,3$)$, enquanto valores altos indicam florestas ou vegetação mais densa $(0,6$ a 0,8$)$, confirmando as classes espectrais (Figura 4).

Em estudo realizado por Bezerra et al. (2018), para a comparação dos dados orbitais pelo processamento de imagens utilizando OLI Landsat $8 \mathrm{e}$ o MSI Sentinel 2, no mesmo período temporal na região do semiárido de Pernambuco, constataram que os valores obtidos do NDVI são correspondentes para os dois satélites no mesmo período de tempo e, assim a classe negativa condiz com esta pesquisa que corresponde aos corpos hídricos da região, em ambos os satélites.

Segundo os estudos de Santiago et al. (2009), foi comprovado que no município de Buíque, inserido na Bacia do Rio Ipanema, valores menores que 0,15 até 0,25 denotando uma região com vegetação estressada ou área degradada pela ação antrópica. Evidenciando assim resultados correlacionados com esta pesquisa. 


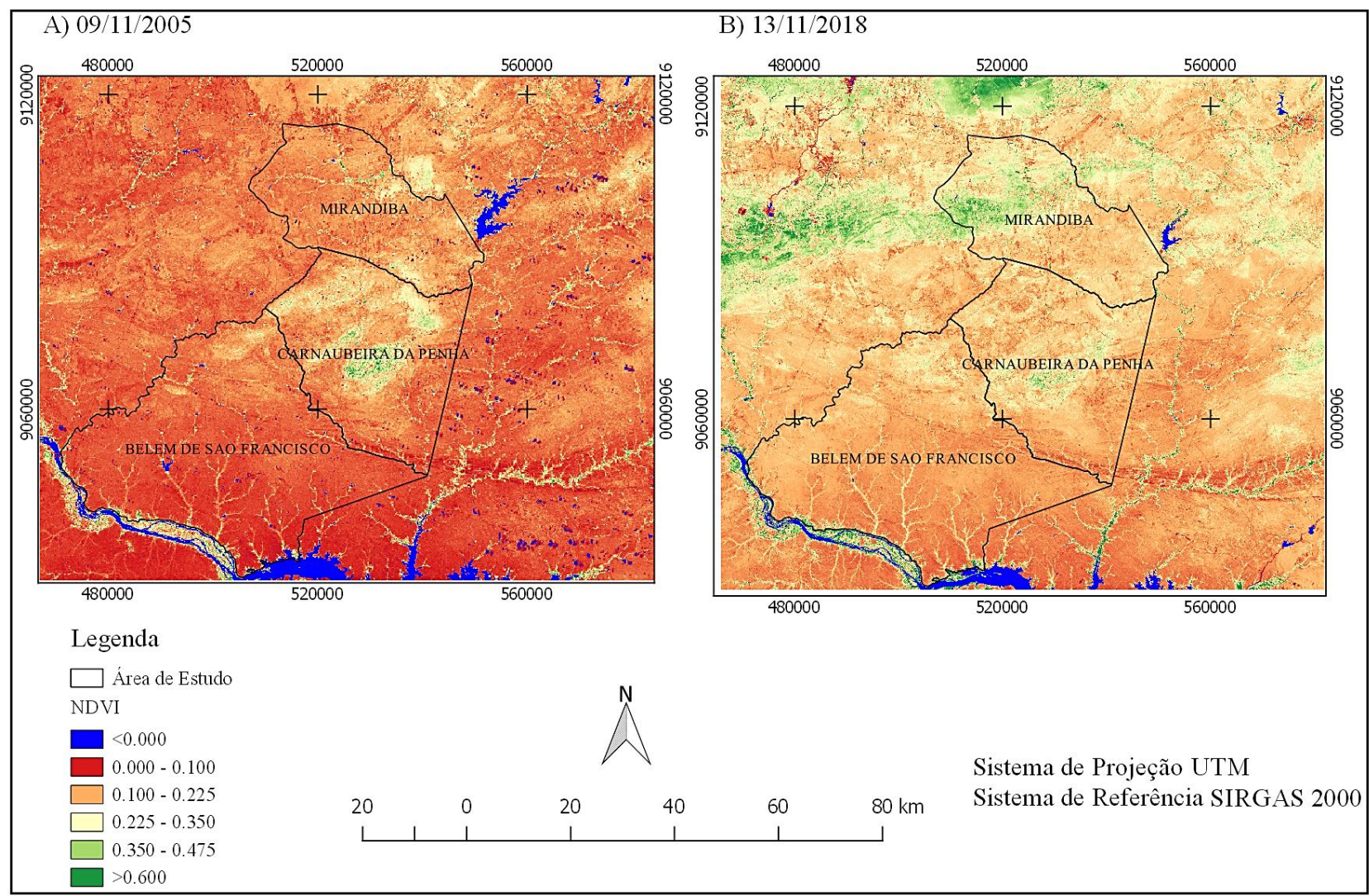

Figura 4 - NDVI da região de estudo nos anos de 2005 e 2018.

Para a imagem referente ao ano de 2005 o NDVI demonstrou valores predominantemente baixos, no intervalo considerado entre 0,0 e 0,1 , tal fator corrobora para afirmação de que por se tratar de uma região do Sertão Nordestino é caracterizada por áreas secas, sendo importante ressaltar que as áreas com valores negativos, representadas pela classe de cor azul (NDVI $<0,0)$, correspondem a regiões onde há corpos hídricos. Como citado anteriormente há a presença de dois importantes corpos hídricos na região, neste estudo foi dado enfoque ao rio Pajeú na região da barragem de Serrinha localizado porção leste do município de Mirandiba.

Nas cartas georreferenciadas as regiões observadas na coloração de tons laranja $(0,0-0,225)$, identificaram-se regiões que em sua predominância possuem valores baixos, porém positivos, correspondente a regiões secas. Para o ano de 2018 observou-se uma redução de regiões de tons vermelho $(0,0-0,10)$, sendo evidenciado pelo aumento no valor do NDVI. Através de uma análise detalhada permitiuse observar que houve uma redução na área correspondente aos corpos hídricos da região, no entanto foi notável que ocorreu um aumento na cobertura vegetal da região.
Observando este fato de uma perspectiva ampla, pode-se afirmar como um dos fatores de causa dessa variação foi a redução da precipitação pluviométrica mensal no ano de 2018 em comparação com os dados do ano de 2005 (Figura 2). Para o ano de 2005, obtivemos um maior volume precipitado no período anterior à data da imagem, volume suficiente para promover o abastecimento dos corpos hídricos da região, aumentando sua área superficial, entretanto a vegetação nativa local é Caatinga, sendo caracterizada por rápidas respostas às precipitações ocorridas. Com base nos dados obtidos no site da APAC, podemos analisar que não ocorreram precipitações pluviométricas nos dois meses anteriores a data da imagem analisada, e o evento de precipitação pluviométrica ocorrido no mês de novembro foi no dia 28 , data posterior ao dia analisado, de maneira que a região estava seca no período estudado.

Porém para o ano de 2018, o volume acumulado de precipitação pluviométrica no período anterior a data da imagem é menor do que no ano de 2005, ocasionando um cenário negativo dos corpos hídricos da região em comparação com o observado no de 2005, porém ocorreram eventos chuvosos no mês de outubro, sendo estes suficientes para proporcionar a 
modificação do cenário da cobertura vegetal da região com uma resposta positiva a vegetação na data da imagem analisada (Figura 4B).
Para uma análise estatística dos dados obtidos, a Tabela 4 demonstra os valores máximos, médios, mínimos e o desvio padrão para cada ano.

Tabela 4 - Dados estatísticos do parâmetro físico NDVI dos anos 2005 e 2018.

\begin{tabular}{ccccc}
\hline Imagem & Máximo & Média & Mínimo & Desvio Padrão (๘) \\
\hline $09 / 11 / 2005$ & 0,780 & 0,184 & $-0,424$ & 0,070 \\
$13 / 11 / 2018$ & 0,777 & 0,284 & $-0,445$ & 0,086 \\
\hline
\end{tabular}

Como detalha a Figura 5, a comparação do perfil longitudinal do NDVI da região do corpo hídrico do rio Pajeú localizado na barragem de Serrinha, referente aos anos 2005 e 2018, sendo possível observar a variação do valor do NDVI, que evidenciou a redução da área do corpo hídrico observado na região, fato já observado e evidenciado na análise comparativa dos valores de NDVI nos anos de estudo. Além de ser possível visualizar a variação do NDVI entre a região referente a cobertura vegetal e a região do rio.

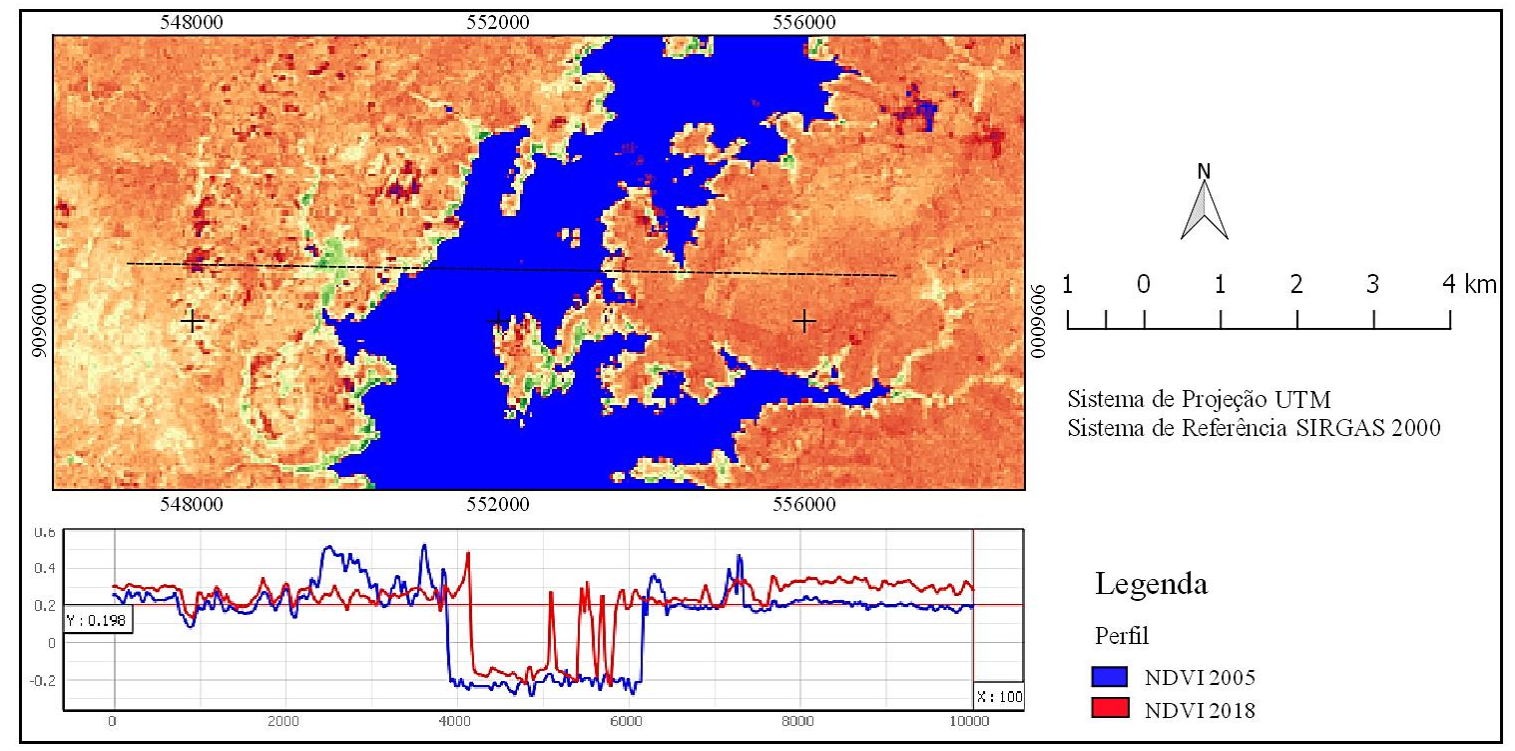

Figura 5 - Perfil Longitudinal georreferenciado do NDVI da região de estudo e do rio Pajeú nos anos de 2005 e 2018.

Na Figura 6, notou-se que os valores obtidos para o NDWI nos anos de estudo, as regiões aver melhadas, referentes aos valores de NDVI $<-0,25$ e no intervalo entre $-0,250$ e $-0,138$ indicaram valores negativos desse índice, sendo realizada uma comparação entre os valores obtidos no ano de 2005 e no ano de 2018. Ferreira et al. (2012) investigaram que para valores de NDWI negativos existe uma correspondência com vegetação seca, solo exposto e áreas urbanas, e valores acima de 0,1 identifica áreas irrigadas com cursos hídricos. Segundo Almeida et al. (2018), as regiões com NDWI $(>0,08)$ foram representadas por áreas com maior presença de água.

Evidenciou-se que ocorreu uma diminuição nos valores máximos obtidos de NDWI no ano de 2018 (Tabela 5), referentes aos valores de NDWI maiores que 0,200 , em comparação com os valores máximos obtidos no ano de 2005, porém foi possível visualizar que houve um aumento na quantidade de áreas irrigadas, fator já caracterizado pelo NDVI, com o aumento nos valores obtidos no ano de 2018, evidenciando a alteração no cenário da cobertura vegetal da região de maneira positiva. 


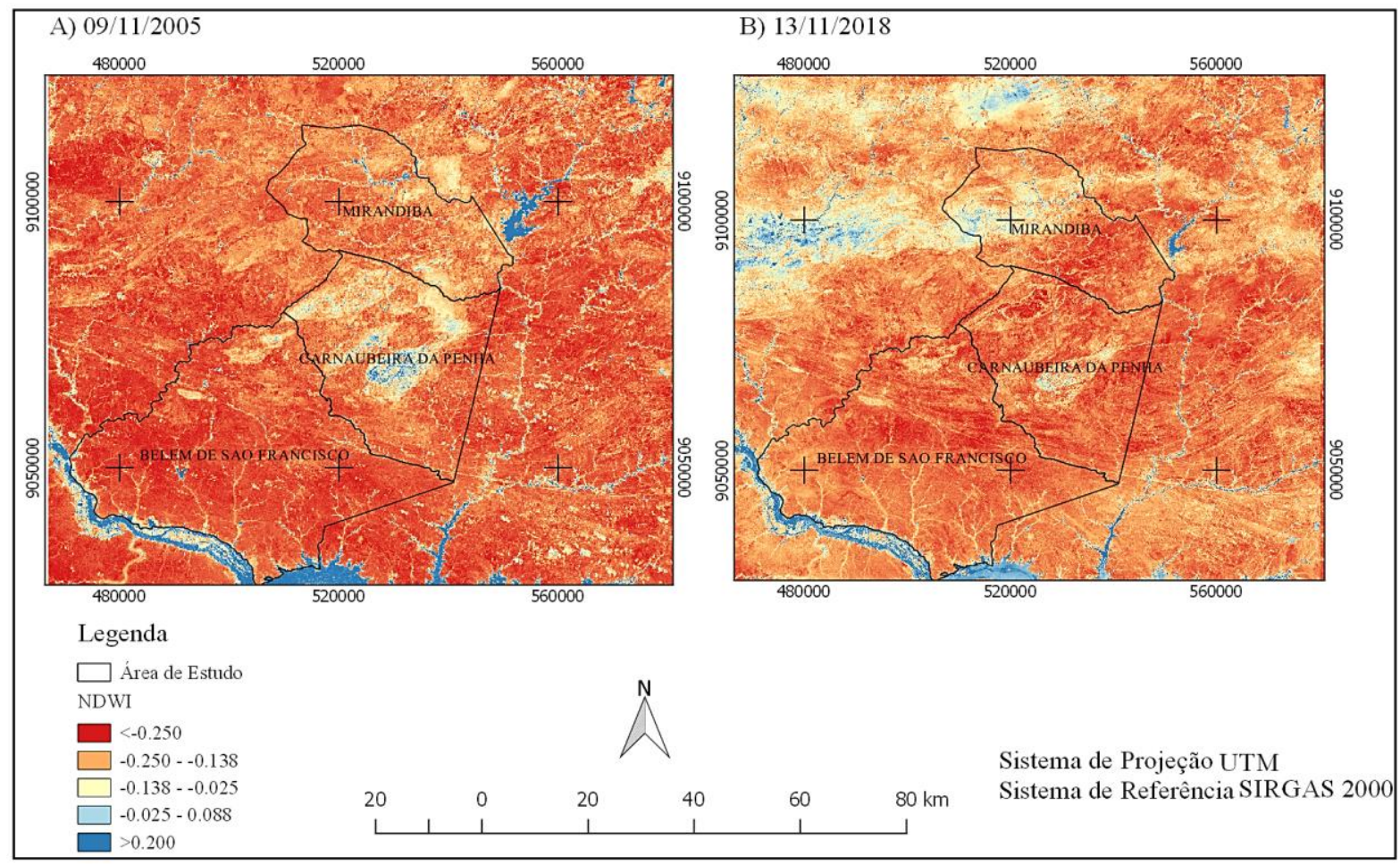

Figura 6. NDWI da região de estudo nos anos de 2005 e 2018.

Em observação aos valores de precipitação pluviométrica na região, visualizou-se um total anual de 799,7 mm para o ano de 2005 e $615 \mathrm{~mm}$ para o ano de 2018, entretanto evidencia-se a explicação anteriormente citada que com relação ao ano de 2005 , não há a ocorrência de eventos chuvosos nos meses de setembro e outubro (Figura 2), período anterior a data da imagem processada, sendo apena evidenciada a ocorrência de precipitação pluviométrica no dia 28 de novembro, enquanto que para o ano de 2018, observam-se períodos secos nos meses de junho a setembro, porém há a ocorrência de eventos chuvosos em poucos dias anteriores a data da imagem analisada.

Portanto, um dos fatores relevantes para a redução dos valores do índice referentes as regiões de corpos hídricos no ano de 2018, foi a diminuição no volume precipitado nos meses anteriores ao dia estudado. Como observado na Figura 6, a região apresentou valores negativos em sua predominância, evidenciando uma região seca, sendo tais resultados corroborados com a conclusão obtida por Leivas et al. (2013), na região da Bahia, apresentando resultados predominantemente inferiores a zero e efetuando a premissa de que tais valores caracterizam uma condição de baixa umidade na vegetação.

A Tabela 5 demonstra a comparação estatísticas dos dados obtidos nos dois anos do NDWI, sendo possível visualizar a variabilidade espectro temporal na região no decorrer dos 13 anos.

Tabela 5. Dados estatísticos do parâmetro físico NDWI dos anos 2005 e 2018.

\begin{tabular}{ccccc}
\hline Imagem & Máximo & Média & Mínimo & Desvio Padrão (๘) \\
\hline $09 / 11 / 2005$ & 0,818 & $-0,163$ & $-0,355$ & 0,081 \\
$13 / 11 / 2018$ & 0,482 & $-0,146$ & $-0,379$ & 0,071 \\
\hline
\end{tabular}

De acordo com estudos realizados na Bacia Hidrográfica do Tapacurá-PE por Oliveira et al. (2013), constatou-se que os valores mais elevados de NDWI representam a vegetação com maior quantidade de água em sua estrutura interna. A partir das conclusões obtidas com o NDWI, foi possível utilizar o valor do índice para a classificação de um corpo hídrico situado na região de estudo, a região da Barragem de Serrinha no rio Pajeú. 
A Figura 7 detalha o perfil longitudinal do NDWI, de maneira a destacar a variabilidade no valor do índice na região de estudo e no corpo hídrico identificado, demonstrando o aumento do valor do índice na região referente ao rio Pajeú em comparação com a área de cobertura vegetal, premissa corroborada pelo estudo desenvolvido por Nascimento et al. (2019) no Semiárido pernambucano. A comparação temporal do NDVI, evidenciou uma diminuição do valor obtido na zona referente ao corpo hídrico para os anos de 2005 e 2018, sendo este fato concluído anteriormente como consequência dos eventos chuvosos ocorridos nos anos de estudo.

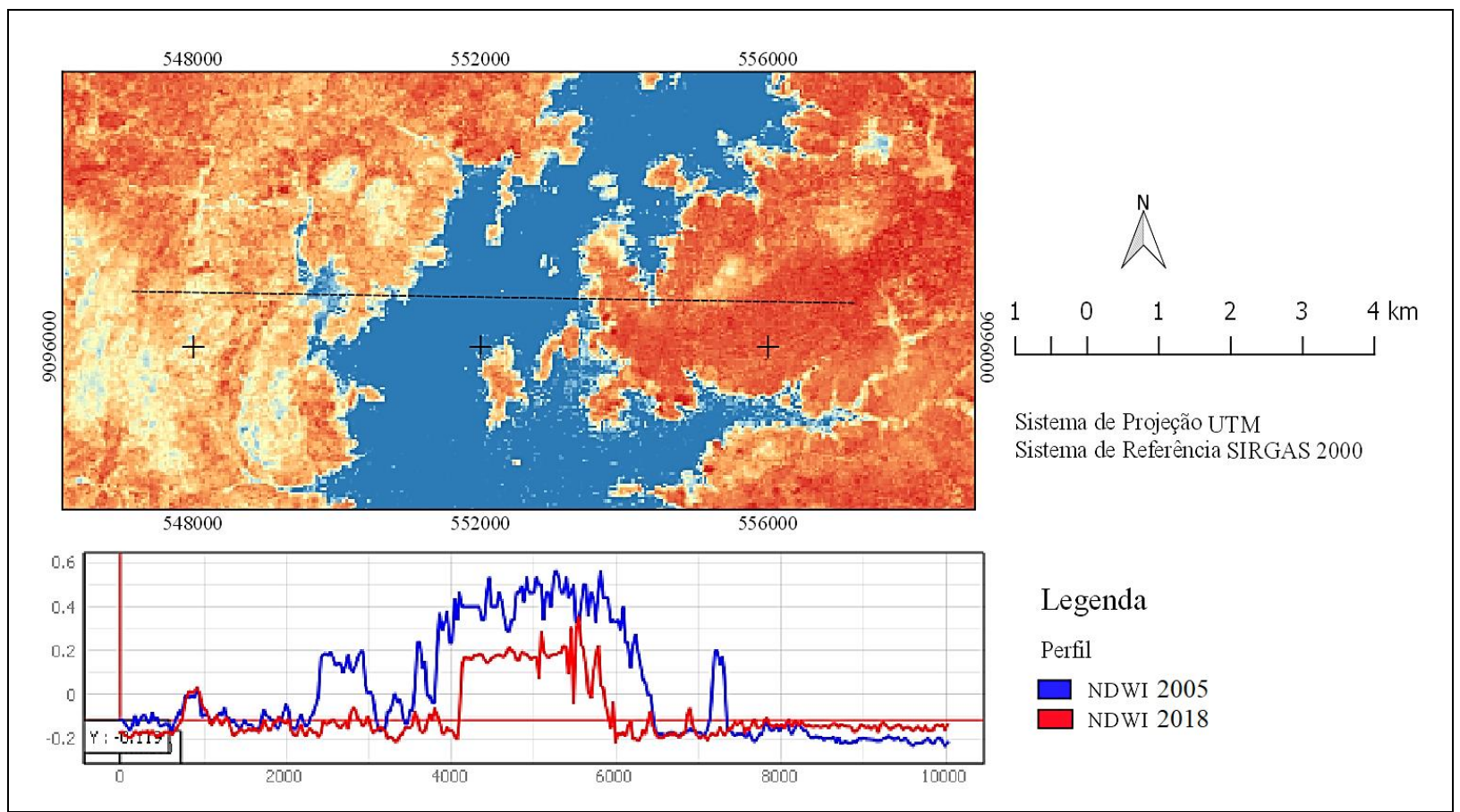

Figura 7 - Perfil Longitudinal georreferenciado do NDWI da região de estudo e no rio Pajeú nos anos de 2005 e 2018.

Aliado aos dados de pluviometria do munícipio, o NDWI, serviu de auxílio para a geração do mapa da área superficial do espelho d'água do rio Pajeú próximo a barragem Serrinha (Figura 8).

$\mathrm{Na}$ Tabela 6, foram determinadas as respectivas áreas superficiais do rio Pajeú através da utilização de geoprocessamento, para isto, foi necessária a vetorização da região que corresponde a feição água extraída a partir do NDWI.

Tabela 6 - Área superficial do rio Pajeú no entorno da barragem Serrinha nos anos 2005 e 2018.

\begin{tabular}{cc}
\hline Ano & Área $\left(\mathrm{m}^{2}\right)$ \\
\hline $09 / 11 / 2005$ & $39.015 .900,00$ \\
$13 / 11 / 2018$ & $27.688 .700,05$ \\
\hline
\end{tabular}

Com a realização da vetorização foi possível o cálculo de área por ferramentas do QGIS. Com isso, verificou-se uma diminuição consideravelmente entre os anos 2005 e 2018 especificamente, uma das razões por este decréscimo foi de uma incidência de seca em 2018 que ocasionou a estiagem.

Pela classificação automatizada adquirida pelo MAPBIOMAS da coleção 3.1, foi possível observar similaridade da interpretação visual que as cartas de NDVI e NDWI demonstraram no que se refere ao trecho do rio Pajeú no entorno a barragem Serrinha.

Como detalha a Figura 9, a classificação de imagens para o ano 2005 houve semelhança na configuração da área superficial do corpo hídrico. Além disso, foi possível avaliar os diversos tipos de classes da cobertura do solo tais como: floresta, uso agropecuário, e cultivo perene conforme a legenda. 


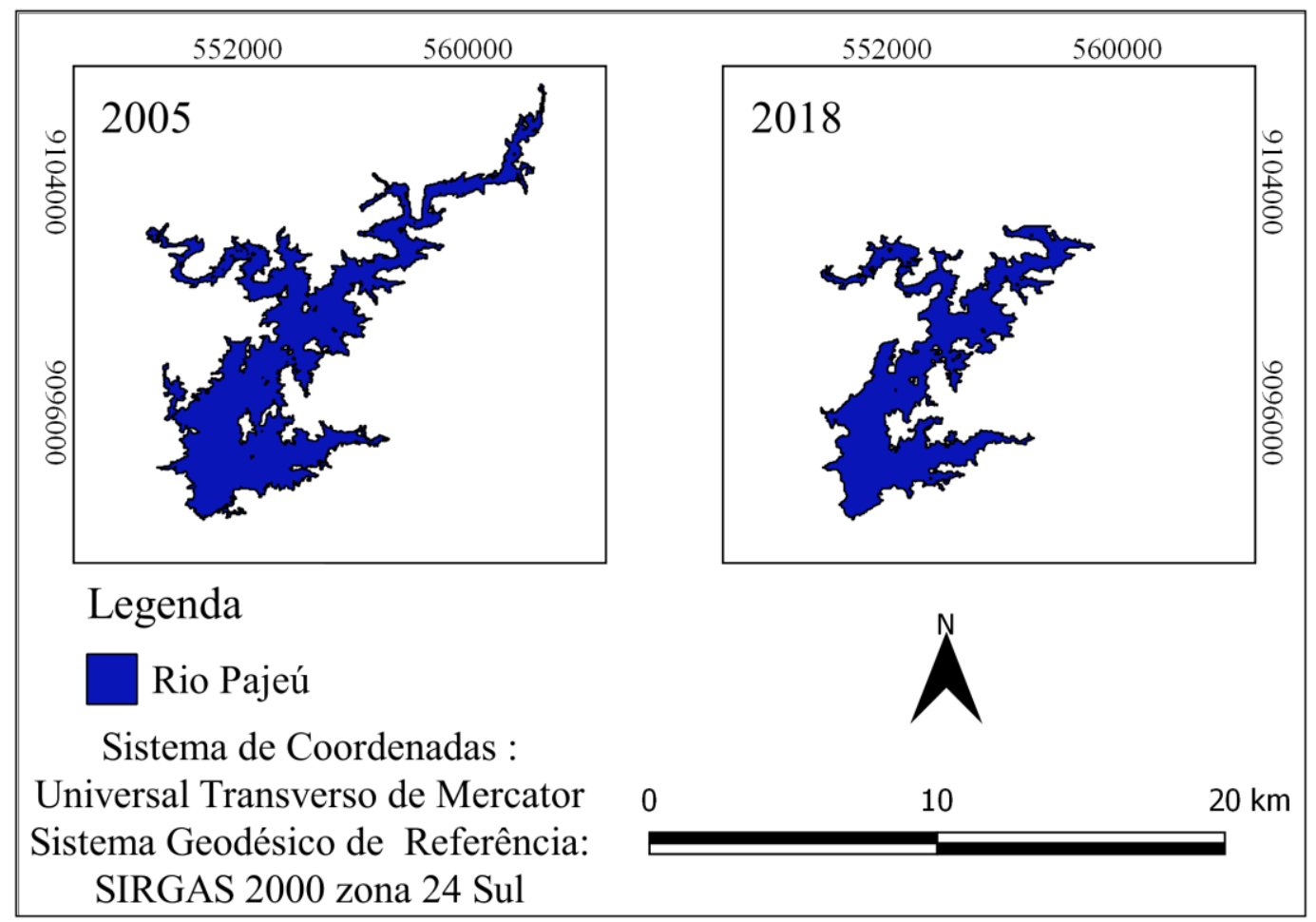

Figura 8 - Área superficial do espelho d'água do reservatório Serrinha nos anos de 2005 e 2018.

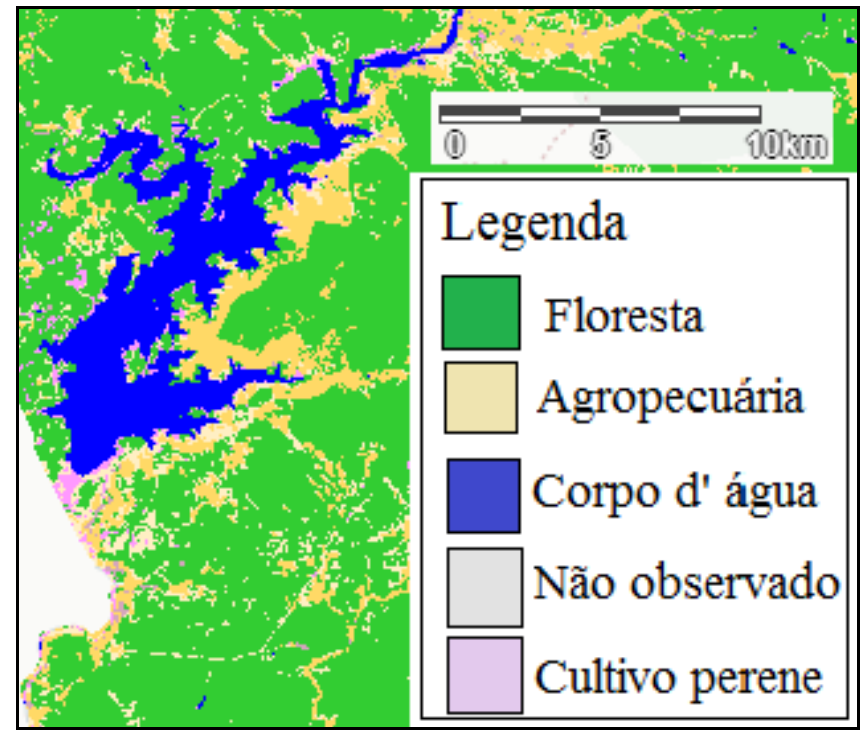

Figura 9 - Classificação automatizada pelo MAPBIOMAS referente ao ano 2005. Fonte: MAPBIOMAS (2019).

Dentre outros dados fornecidos pelo MAPBIOMAS coleção 3.1, tem-se os gráficos gerados automaticamente apresentando a área de cada classe selecionada configurada na área de interesse. Como demonstra a Figura 10, os corpos hídricos presentes no município de Serra Talhada, onde está inserida a barragem Serrinha, tiverem consideravelmente redução ao longo dos anos. Este gráfico demonstra esse comportamento do ano 1985 até 2017. No ano 2005, a área dos corpos hídricos em geral atingiu 4.269,63 hectares. 


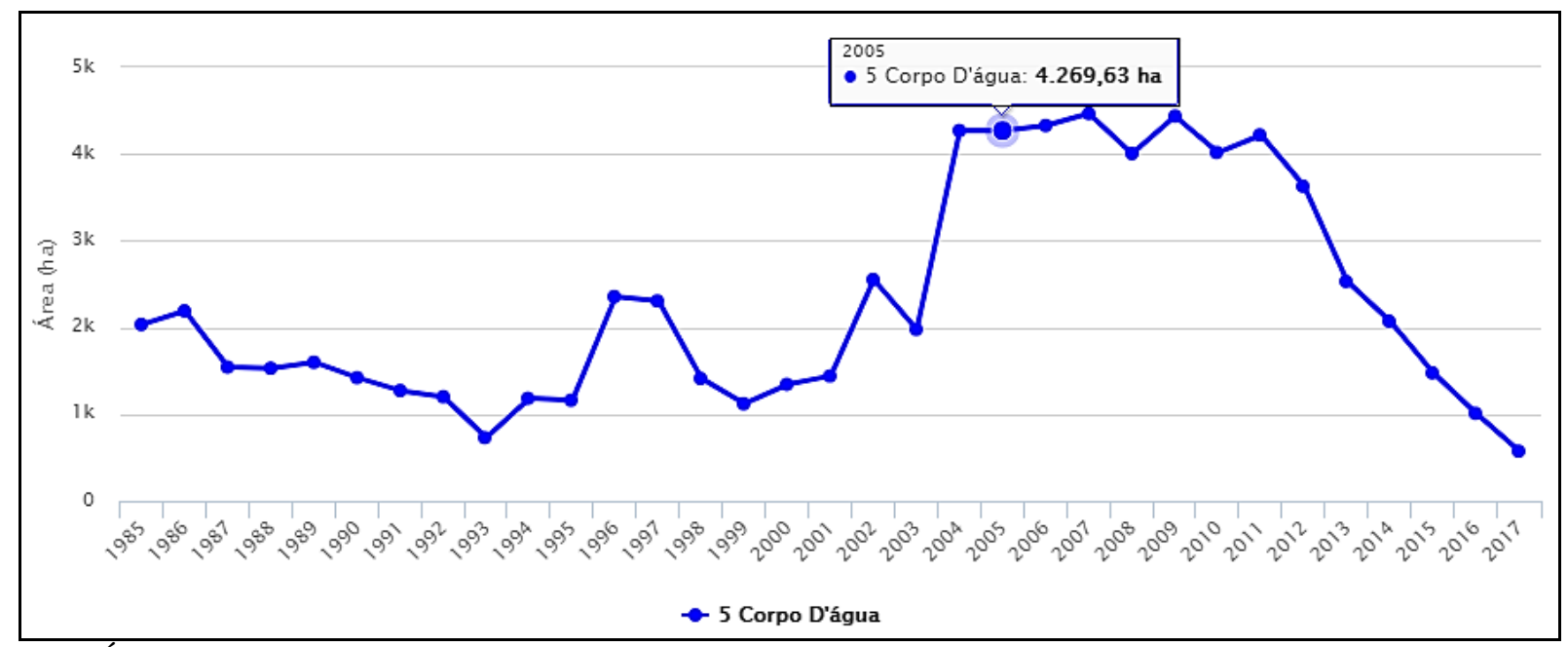

Figura 10 - Área superficial de corpos hídricos em Serra Talhada ao longo dos anos entre 1985 a 2017. Fonte: MAPBIOMAS (2019).

\section{Conclusões}

As ferramentas utilizadas para este trabalho, os índices NDVI, NDWI e a plataforma MAPBIOMAS coleção 3.1 permitiram resultados semelhantes para estudos da cobertura e uso do solo realizado na área de estudo.

Os índices estudados nesse artigo para os municípios de Mirandiba, Carnaubeira da Penha e Belém de São Francisco, evidenciaram a importância da identificação e análise da cobertura e uso da superfície do solo, com a praticidade e precisão devido ao uso de imagens georreferenciadas e estudadas no software livre QGIS.

Conforme foram analisados os índices, concluiu-se que o NDVI apontou uma vegetação estressada para o ano de 2005, e uma variação da cobertura com um aumento da vegetação ao longo dos 13 anos de estudos até o ano de 2018.

O NDWI apontou valores de vegetação seca e solo exposto em grande parte da região para o ano de 2005, e isso é evidenciado também pela data da imagem do TM Landsat 5, que foi obtida num período de baixos índices pluviométricos, porém na comparação temporal, podemos observar que houve aumento no valor desse índice para o ano de $2018 \mathrm{em}$ sua predominância, apesar de demonstrar uma redução das áreas correspondentes aos corpos hídricos para este ano, fator explicado pelo ausência de precipitação pluviométrica na data da imagem que possibilitasse a mudança no cenário do corpo hídrico.

\section{Agradecimentos}

Os autores agradecem ao Conselho Nacional de Desenvolvimento Científico e Tecnológico (CNPQ) pelo incentivo a pesquisa e concessão de bolsa de iniciação científica da primeira e terceira autoras, a Coordenação de Aperfeiçoamento de Pessoal de Nível Superior (CAPES) pelo incentivo a pesquisa e concessão de bolsa de Pós-Graduação da quarta autora; ao United States Geological Survey (USGS) pela aquisição da imagem de satélite; a Agência Pernambucana de Águas e Clima (APAC) pelo banco de dados hidrológicos e ao Projeto de Pesquisa intitulado "Coberturas vegetal e hídrica de bacias hidrográficas utilizando imagens orbitais no estado de Pernambuco", aprovado na Chamada Universal MCTIC/CNPq 2018 - FAIXA A, processo $\mathrm{n}^{\circ}$ 433914/2018-1, da segunda autora.

\section{Referências}

Allen, R.G., Waters, R.; Trezza, R., Tasumi, M., Bastiaanssen, W., 2002. Sebal - Surface Energy Balance Algorithms for Land: Idaho implementation: advance training and users manual. Idaho Department of Water Resources, Moscow.

Almeida, D.N.O., Oliveira, L.M.M., Candeias, A.L.B., Nascimento, E.F., Le,ite, A.C.S., 2018. Sensoriamento Remoto do ambiente hídrico: Índice de umidade (NDWI) no Açude Poço da Cruz - PE. Meio Ambiente em Foco, Recife.

Almeida, D.N.O., Oliveira, L.M.M., Candeias, A.L.B., Bezerra, U.A., Leite, A.C.S., 2018. Uso e cobertura do solo utilizando geoprocessamento em municípios do Agreste de Pernambuco. Revista Brasileira de Meio Ambiente 4, 58-68. 
Andrade, C.B., Omena, J.A.M., Villar, A.C., Gusmão, L., Rodrigues, D.F.B., Oliveira, L.M.M., 2018. Avaliação de índices de vegetação e características fisiográficas no Sertão Pernambucano. Revista Brasileira de Meio Ambiente 4, 97-107.

APAC. Agência Pernambucana de Águas e Climas, 2019. Sistema de Informação Geográfica. Disponível: http://www.apac.pe.gov.br/. Acesso: 1 nov. 2019.

Araújo, F.E., Anjos, R.D., Candeias, A.L.B., Filho, G.B.R., 2015. Levantamento de áreas verdes no campus da UFPE com uso de geoprocessamento. Workshop de Mudanças Climáticas e Recursos Hídricos do Estado de Pernambuco. Recife.

Bezerra, U.A., Oliveira, L.M.M., Candeias, A.L. B., Silva, B.B., Leite, A.C.L.S., Silva, L.T.M.S., 2018. Comparativo do Índice de Vegetação de Diferença Normalizada (NDVI) entre os Sensores OLI Satélite Landsat-8 e MSI - Satélite Sentinel-2 em Região Semiárida. Anuário do Instituto de Geociências - UFRJ. Disponível: www.anuario.igeo.ufrj.br. Acesso: 6 jun. 2019.

Chouhan, R., Rao, N., 2011. Vegetation detection in multispectral remote sensing images: Protective role-analysis of vegetation in 2004 indian ocean tsunami. Geo-Information for disaster management, Turkey. Disponível: http://www.isprs.org/proceedings/2011/Gi4DM/PD F/OP37.pdf. Acesso: 6 jun. 2019.

Farias, M.O., Candeias, A.L.B., Oliveira, L.M.M., Almeida, D.N.O., Tavares Junior, J.R., 2017. Variabilidade espaço-temporal da cobertura do solo por índices de vegetação e água no Agreste Pernambucano. Revista Brasileira de Agrotecnologia 7, 91-99.

Ferreira, J.M.S., Ferreira, H.S., Silva, H.A., Santos, A.M., Galvíncio, J.D., 2012. Análise espaçotemporal da dinâmica da vegetação da caatinga no município de Petrolina-PE. Revista Brasileira de Geografia Física 5, 904-922.

Gao, B., 1996. NDWI - a Normalized Difference Water Index for remote sensing of vegetation liquid water from space. Remote Sensing of Environment 58, 257-266.

Gomes, S.O., Lima, V.N., Candeias, A.L.B., Silva, R.R., 2017. Uso e cobertura dos solos de Petrolândia utilizando MAPBIOMAS. Congresso Brasileiro de Cartografia. Rio de Janeiro.

Gonzaga, E.P., Santos, V.V., Nicácio, R.M., 2011. Análise do comportamento do NDVI e NDWI em períodos de diferentes intensidades pluviométricas no Sertão alagoano. Simpósio Brasileiro de Sensoriamento Remoto. Curitiba.
Gordon, L.J., Steffen, W., Jönsson, B.F., Folke, C., Falkenmark, M., Johannesen, А., 2005. Human modification of global water vapor flows from the land surface. Proceedings of the National Academy of Sciences 102, 7612-7617.

Leivas, J.F., Andrade, R.G., Vicente, L.E., Torresan, F.E., Victoria, D.C., Bolfe, E.L., 2013. Monitoramento da seca de 2011/12 a partir do NDWI e NDVI padronizado do SPOT-Vegetation. Simpósio Brasileiro de Sensoriamento Remoto. Foz do Iguaçu.

Lima, V.N., Gomes, S.O., Candeias, A.L.B., Silva, R.R., 2017. MAPBIOMAS e uso e cobertura dos solos do município de Brejinho, Pernambuco. Congresso Brasileiro de Cartografia. Rio de Janeiro.

Markham, B.L., Barker, J.L., 1987. Thematic Mapper bandpass solar exoatmospheric irradiances. International Journal of Remote Sensing 8, 517-523.

Nascimento, E.F., Oliveira, L.M.M., Lima. J.F., Farias, A.A.M., Silva, J.G., 2019. Comparação de índices de água na identificação de corpo hídrico por sensoriamento remote. Simpósio Brasileiro de Sensoriamento Remoto. Santos.

Oliveira, L.M.M., Montenegro, S.M.G.L., Silva, B.B., Moura, A.E.S.S., Gusmao, A.C.V.E.L., Salgueiro, J.H.P.B., 2013. Índices de vegetação por sensoriamento remoto na bacia hidrográfica do rio Tapacurá - PE. Simpósio Brasileiro de Recursos Hídricos. Bento Gonçalves.

Ponzoni, F.J., 2002. Sensoriamento Remoto no Estudo da Vegetação: diagnosticando a mata atlântica. INPE, São José dos Campos.

PROJETO MAPBIOMAS - Coleção 3.1 da Série Anual de Mapas de Cobertura e Uso de Solo do Brasil. Disponível: http://mapbiomas.org. Acesso: 22 jun. 2019.

Rouse, J.W., Haas, R.H., Schell, J.A., Deering, D.W., 1973. Monitoring vegetation systems in the Great Plains with ERTS. In: Earth Resources Technology Satellite-1 Symposium. Washington.

Santiago, M.M., Silva, H.A., Galvincio, J.D., Oliveira, T.H., 2009. Análise da Cobertura Vegetal Através dos Índices de Vegetação (NDVI, SAVI e IAF) no Entorno da Barragem do Botafogo-PE. Simpósio Brasileiro de Sensoriamento Remoto. Natal.

Silva, J.G., Oliveira, L.M.M., Nascimento, E.F., 2019. Análise temporal da cobertura do solo por índices de vegetação no município de Belo Jardim - PE. Simpósio Brasileiro de Sensoriamento Remoto. Natal. 